\title{
Landing Risk Evaluation of Carrier-Based Aircraft Based on Mean-Variance Statistics Error Multi-Attribute Dynamic Decision Making
}

\author{
Li Hui \\ School of Computer and Information Engineering \\ Harbin University of Commerce \\ hrbcu_lh@163.com
}

\begin{abstract}
To induce the landing risk of carrier-based aircraft, and improve the safety of pilots and carrier-based aircraft, this paper presents an improved landing risk evaluation of carrier-based aircraft based on Mean-Variance statistics error multi-attribute dynamic decision making. Attribute values is shown as triangular fuzzy numbers, and ordering vectors are achieved through possibility-degree and ordering expression. In accordance with ordering wave from ordering values at different times to desire rank, condition analysis should be conducted. Finally, the rank ordering is acquired. The model simulation results indicate the better performance of the new method in comparison with the traditional controller with more accuracy and practicability.
\end{abstract}

Keywords: Mean-Variance Statistics Error; Multi-Attribute Dynamic Decision Making; Landing Risk Evaluation; Carrier-Based Aircraft

\section{Introduction}

Landing risk of carrier-based aircraft has always existed in every point during landing process. To ensure landing safely and quickly, there are lots of studies for researching. [1] had established the Landing Signal Officer (LSO) instruction associated with operation guide system to control hazard. Asymmetric variable universe adaptive landing fuzzy controller for carrier-based aircraft was designed to control the landing voyage in [2]. Integrated evaluation technology of LSO should be presented for assessing landing risk in [3].

Dynamic Multi-Attribute Decision Making (DMADM) based on advantage retention degree is described in [4]. An improved VIKOR should be presented for researching triangular fuzzy numbers in [5]. Landing risk assessment based on fuzzy multi-attribute decision making should be discussed in [6]. It is used concentrated factors to amalgamate the decision making information at different time points for the traditional dynamic decision problems, as that we call the dynamic decision making above is another Group Multi-Attribute Decision Making (GMADM).

Considering landing of carrier-based aircraft is a process from the start position to the desire point, every decision time has connection with each other. Landing risk evaluation of carrier-based aircraft based on Mean-Variance statistics error multi-attribute decision making should be proposed in this paper.

The rest of this paper is structured as follows: next section we first analyze the landing risk evaluation problem. Section 3 designs the landing risk evaluation of carrier-based aircraft based on Mean-Variance statistics error multi-attribute dynamic decision making. Multiple landing simulations of carrier-based aircraft should be shown in Section 4. 


\section{Problem Description}

Generally speaking, for landing system of carrier-based aircraft which is shown in Figure. 1, landing risk evaluation should be realized as Figure. 2 by four steps [9-11]:

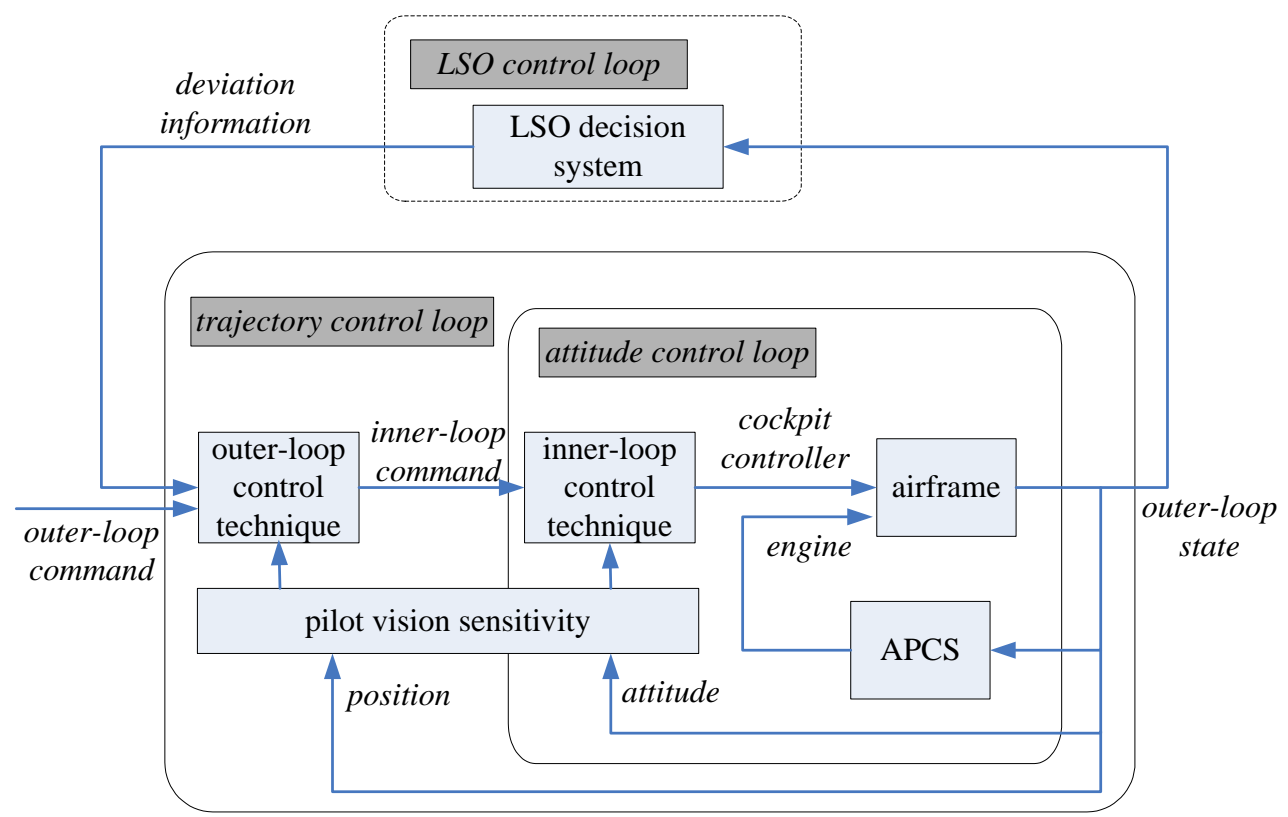

Figure 1. Landing system of Carrier-Based Aircraft

1) Explicit four evaluation positions: start position(X), middle position(IM), close position(IC) and ramp position(AR).

2) Obtain decision making records of flight status information at different positions.

3) Make certain discrete time of four standard positions.

4) Dynamic decision making with decision information using appropriate ways.

Dynamic decision making for carrier-based aircraft is the correlative problem in allusion to the discrete time points. Flight status will be represented as nine criterion description, and time moments information should be interacted for final alternatives ranking order. 


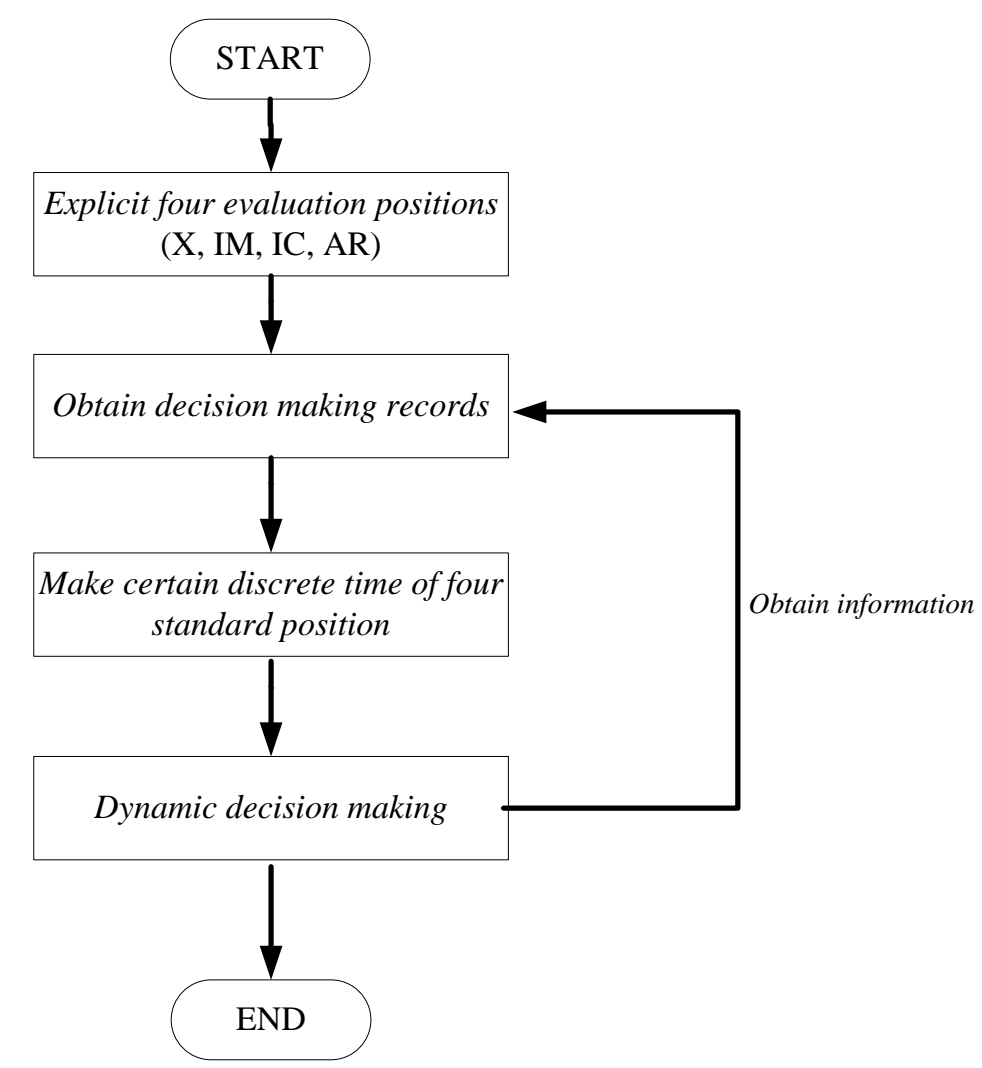

Figure 2. Landing Risk Evaluation Steps

\section{Landing Risk Evaluation of Carrier-Based Aircraft Based on Mean- Variance Statistics Error Multi-Attribute Dynamic Decision Making}

Landing risk evaluation is an complicated process. For one dynamic decision making problem, outset let $\boldsymbol{X}=\left\{x_{1}, x_{2}, \ldots x_{m}\right\}$ be a discrete set of $m$ decision feasible alternatives, $\boldsymbol{U}=\left\{u_{1}, u_{2}, \ldots u_{n}\right\}$ is a finite set of attributes, and $\boldsymbol{T}=\left\{t_{1}, t_{2}, \ldots t_{l}\right\}$ is a set of decision time points. Construct $\boldsymbol{R}^{k}=\left(r_{i j}^{(k)}\right)_{m \times n}$ a decision matrix as shown in (1) [12-15].

$\begin{array}{llll}u_{1} & u_{2} & \ldots \ldots & u_{n}\end{array}$

$\boldsymbol{R}_{\boldsymbol{k}}=\left[\begin{array}{cccc}r_{11}^{(k)} & r_{12}^{(k)} & \ldots & r_{1 n}^{(k)} \\ r_{21}^{(k)} & r_{22}^{(k)} & \ldots & r_{2 n}^{(k)} \\ \ldots & \ldots & \ldots & \ldots \\ r_{m 1}^{(k)} & r_{m 2}^{(k)} & \ldots & r_{m n}^{(k)}\end{array}\right] \quad \begin{gathered}x_{1} \\ x_{2} \\ \ldots \\ x_{m}\end{gathered}$

The steps of landing risk evaluation based on Mean-Variance statistics error multiattribute decision making are:

Step 1: Explicit attributes $\boldsymbol{\omega}=\left(\omega_{1}, \omega_{2}, \ldots \omega_{m}\right)^{\mathrm{T}}$. As landing assessment hierarchy in [4], there are three influencing indicator during lading loop: glideslope deviation, speed and rate of decent (ROD). As we know, glideslope deviation and speed are main risk indicator, the other one is lesser. Let attributes are defined as (2): 
$\omega_{j}=\left\{\begin{array}{cc}\frac{1-\lambda}{n}+\frac{\lambda}{n-1}, & j \neq n \\ \frac{1-\lambda}{n}, & j=n\end{array}\right.$.

where $n=3, \lambda=0.2$ and $\omega=(0.4,0.4,0.2)^{\mathrm{T}}$.

Step 2: Calculate comprehensive attribute evaluation value at $t_{k}$ moment. The ${ }_{i}(i \in M)$ th column data of matrix $R^{k}$ should be concentrated using TFLWA operator, and comprehensive attribute evaluation value of $x_{i}$ at $t_{k}$ moment is:

$z_{i}^{k}=\sum_{j=1}^{n} \omega_{j} r_{i j}^{k}$.

Step 3: Install possibility-degree matrix at every moment as possibility-degree formula of triangle fuzzy numbers [16-18].

$p_{i j}^{k}=p\left(z_{i}^{k} \geq z_{j}^{k}\right)(i, j \in M)$.

Step 4: Acquire ordering vector $\omega^{k}=\left(\omega_{1}, \omega_{2}, \ldots, \omega_{m}\right)$ at $t_{k}$ point by ordering formula [19-22].

$$
\omega_{i}=\frac{1}{n(n-1)}\left(\sum_{j=1}^{n} p_{i j}+\frac{n}{2}-1\right) .
$$

Step 5: Confirm attribute $\varpi=\left(\varpi_{1}, \varpi_{2}, \ldots, \varpi_{l}\right)^{\mathrm{T}}$ of time with four glideslope standard positions as dynamic decision making time. From [6], we know that the farer to carrier, the larger for safe flight area, and the less risk for pilots. So the relative attribute weight relationship of standard positions is shown as (6).

$\varpi_{\mathrm{X}}<\varpi_{\mathrm{IM}}<\varpi_{\mathrm{IC}}<\varpi_{\mathrm{AR}}$

where we can describe as (7).

$$
\varpi_{i}=\frac{1}{z_{p_{i}}^{u p}-z_{p_{i}}^{d o w n}} \quad(i=\mathrm{X} 、 \mathrm{IM} 、 \mathrm{IC} \text { or } \mathrm{AR}) .
$$

Finally,

$\varpi=\left(\varpi_{\mathrm{X}}, \varpi_{\mathrm{IM}}, \varpi_{\mathrm{IC}}, \varpi_{\mathrm{AR}}\right)=(0.0403,0.0638,0.1231,0.7728)^{\mathrm{T}}$.

Step 6: Calculate expected rank value of $x_{i}$. The expected value should be gained by rank value of $x_{i}$ at different time points, and expected rank value of $x_{i}$ is shown as (9).

$$
E_{x_{i}}=\sum_{k=1}^{l} \varpi_{k} w_{i}^{k} \text {. }
$$

Step 7: Ascertain discrete deviation of $x_{i}$. Because of various kinds of elements, there is certain deviation between rank value of $x_{i}$ at different moments and expected rank $E_{x i}$ value of $x_{i}$, and variance $\sigma_{i}^{2}(k)=\left(w_{i}^{k}-E_{i}\right)^{2}$ should be used to express the deviation at definite moment. $\sigma_{i}^{2}(k)$ is the influence of expected rank value of $x_{i}$ by rank value at $k$ moment, and the risk is larger with $\sigma_{i}^{2}(k)$ is more. 
To indicate general fluctuation of the entire alternative $x_{i}$, the rank values are related to seek the general deviation of expeceted $x_{i}$ at different moments, recording discrete deviation of $x_{i}$.

$$
\sigma_{i}^{2}=\sum_{k=1}^{l} \sigma_{i}^{2}(k)=\sum_{k=1}^{l}\left(w_{i}^{k}-E_{i}\right)^{2}
$$

With $\sigma_{i}^{2}$ is more, the landing risk is larger, and the stability is less relatively. Finally flight evaluation effect is minor of voyages $x_{i}$.

Step 8: Decision analysis based on Mean-Variance statistics error. Rank the $m$ expected rank value and discrete deviations of alternatives according value. The sequence of mean is positive, and the ordering position is frontier with mean larger, from 1 to $\mathrm{m}$. On the contrary, the sequence of variance is reverse, and the ordering position is frontier with variance less, from 1 to $m$ similarly. We can execute rank estimation as below situations.

1) If the ordering result of mean is identical of variance's, no difference, and we establish the alternatives sequence as the ordering result of mean and variance.

2) If the ordering result of mean is different of variance's, we should establish the alternatives sequence as (11).

$$
E_{i}-E_{j}\{\geq o r<\} \xi \cdot \frac{1}{m}\left(E_{i}>E_{j}\right)
$$

where $\xi$ is the accuracy of alternatives estimation, and $\frac{1}{m}$ is the equivalent intermission span of alternatives.

$$
\begin{aligned}
& \text { If } E_{i}-E_{j}>\xi \cdot \frac{1}{m} \text {, we install the sequence as the ordering result of mean; } \\
& \text { If } E_{i}-E_{j}<\xi \cdot \frac{1}{m} \text {, we install the sequence as the order result of variance. }
\end{aligned}
$$

\section{Model Simulation}

Five times landing processes of carrier-based aircraft are simulated for analysis, and final distributions of touchdown points are shown in Figure. 3. Landing risk decision making is realized as follow.

There are five flight voyages as alternatives $X=\left[x_{1}, x_{2}, x_{3}, x_{4}, x_{5}\right]$, and risk index of linguistic loop as attributes $U=$ [glideslope deviation, velocity, rate of descend]= $\left[u_{1}, u_{2}, u_{3}\right]$, four standard positions of landing process as discrete decision making time $T=\left[t_{1}, t_{2}, t_{3}, t_{4}\right]$, respectively, the decision matrices $R^{k}=\left(r_{i j}^{k}\right)_{m \times n}$ $(k=1,2,3,4 ; i=1,2,3,4,5 ; j=1,2,3)$ here as listed in Tables $1-4$. 


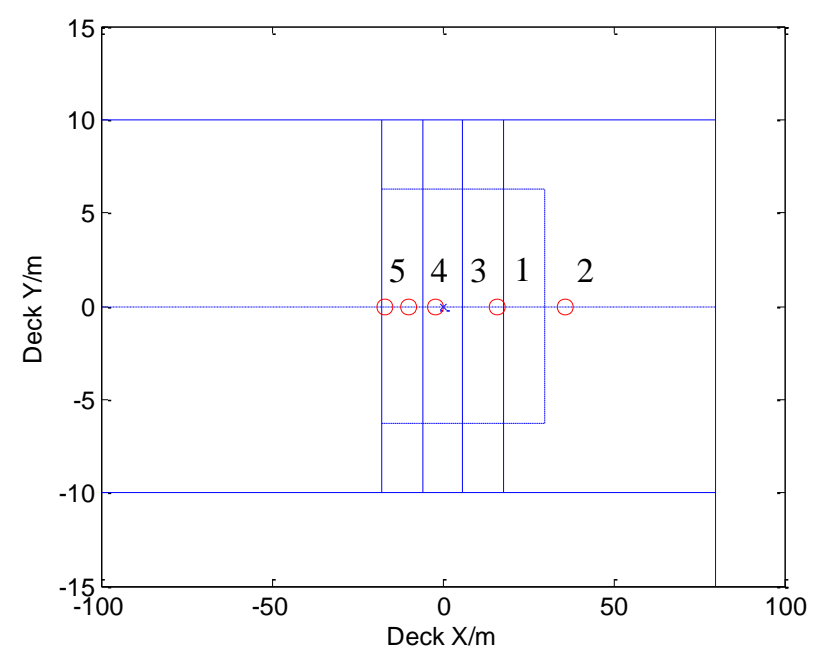

Figure 3. Distributions of Touchdown Points

Table 1. Evaluation Matrix of Decision Moment $\mathrm{t1}$ (X point)

\begin{tabular}{cccc}
\hline Pilotlflight state & $u_{1}$ & $u_{2}$ & $u_{3}$ \\
\hline$x_{1}$ & {$[0.2,0.3,0.4]$} & {$[0.1,0.2,0.3]$} & {$[0.1,0.2,0.3]$} \\
$x_{2}$ & {$[0.1,0.2,0.3]$} & {$[0.3,0.4,0.5]$} & {$[0.2,0.3,0.4]$} \\
$x_{3}$ & {$[0.5,0.6,0.7]$} & {$[0.6,0.7,0.8]$} & {$[0.5,0.6,0.7]$} \\
$x_{4}$ & {$[0.3,0.4,0.5]$} & {$[0.4,0.5,0.6]$} & {$[0.3,0.4,0.5]$} \\
$x_{5}$ & {$[0.3,0.4,0.5]$} & {$[0.1,0.2,0.3]$} & {$[0.1,0.2,0.3]$} \\
\hline
\end{tabular}

Table 2. Evaluation Matrix of Decision Moment t2 (IM point)

\begin{tabular}{cccc}
\hline Pilottflight state & $u_{1}$ & $u_{2}$ & $u_{3}$ \\
\hline$x_{1}$ & {$[0.3,0.4,0.5]$} & {$[0.3,0.4,0.5]$} & {$[0.2,0.3,0.4]$} \\
$x_{2}$ & {$[0.2,0.3,0.4]$} & {$[0.3,0.4,0.5]$} & {$[0.3,0.4,0.5]$} \\
$x_{3}$ & {$[0.6,0.7,0.8]$} & {$[0.7,0.8,0.9]$} & {$[0.6,0.7,0.8]$} \\
$x_{4}$ & {$[0.4,0.5,0.6]$} & {$[0.4,0.5,0.6]$} & {$[0.4,0.5,0.6]$} \\
$x_{5}$ & {$[0.4,0.5,0.6]$} & {$[0.1,0.2,0.3]$} & {$[0.2,0.3,0.4]$} \\
\hline
\end{tabular}

Table 3. Evaluation Matrix of Decision Moment $t 3$ (IC point)

\begin{tabular}{cccc}
\hline Pilotlflight state & $u_{1}$ & $u_{2}$ & $u_{3}$ \\
\hline$x_{1}$ & {$[0.4,0.5,0.6]$} & {$[0.3,0.4,0.5]$} & {$[0.3,0.4,0.5]$} \\
$x_{2}$ & {$[0.3,0.4,0.5]$} & {$[0.4,0.5,0.6]$} & {$[0.3,0.4,0.5]$} \\
$x_{3}$ & {$[0.7,0.8,0.9]$} & {$[0.7,0.8,0.9]$} & {$[0.7,0.8,0.9]$} \\
$x_{4}$ & {$[0.5,0.6,0.7]$} & {$[0.5,0.6,0.7]$} & {$[0.6,0.7,0.8]$} \\
$x_{5}$ & {$[0.5,0.6,0.7]$} & {$[0.3,0.4,0.5]$} & {$[0.3,0.4,0.5]$} \\
\hline
\end{tabular}

Table 4. Evaluation Matrix of Decision Moment t4 (AR point)

\begin{tabular}{cccc}
\hline Pilotfflight state & $u_{1}$ & $u_{2}$ & $u_{3}$ \\
\hline$x_{1}$ & {$[0.5,0.6,0.7]$} & {$[0.5,0.6,0.7]$} & {$[0.4,0.5,0.6]$} \\
$x_{2}$ & {$[0.4,0.5,0.6]$} & {$[0.5,0.6,0.7]$} & {$[0.4,0.5,0.6]$} \\
$x_{3}$ & {$[0.8,0.9,1.0]$} & {$[0.8,0.9,1.0]$} & {$[0.7,0.8,0.9]$} \\
$x_{4}$ & {$[0.7,0.8,0.9]$} & {$[0.6,0.7,0.8]$} & {$[0.7,0.8,0.9]$} \\
$x_{5}$ & {$[0.6,0.7,0.8]$} & {$[0.4,0.5,0.6]$} & {$[0.4,0.5,0.6]$} \\
\hline
\end{tabular}


(1) Use the TFLWA operator to aggregate the $i^{(i \in M)}$ th column data of matrix $R^{1}$, and compute the comprehensive attribute value at $t_{1}$ moment:

$$
\begin{gathered}
z_{1}^{1}=[0.14,0.24,0.34], z_{2}^{1}=[0.2 .0 .3 .0 .4], z_{3}^{1}=[0.54,0.64,0.74], \\
z_{4}^{1}=[0.34,0.44,0.54], \quad z_{5}^{1}=[0.18,0.28,0.38] .
\end{gathered}
$$

(2) Calculate the possibility-degree at $t_{1}$ moment, and construct the possibility-degree matrix $P^{1}=\left(p^{1}\right)_{m \times m}$.

$$
P^{1}=\left[\begin{array}{ccccc}
0.5 & 0.245 & 0 & 0 & 0.32 \\
0.755 & 0.5 & 0 & 0.045 & 0.595 \\
1 & 1 & 0.5 & 1 & 1 \\
1 & 0.955 & 0 & 0.5 & 0.98 \\
0.68 & 0.405 & 0 & 0.02 & 0.5
\end{array}\right]
$$

(3) Compute ordering vector $\omega^{1}$ at $t_{1}$ moment.

$$
\omega^{1}=(0.1283,0.1697,0.3,0.2468,0.1553)^{\mathrm{T}}
$$

Similarly, repeat (1)-(3), we can obtain ordering vectors at other moments.

$$
\begin{gathered}
\omega^{2}=(0.1678,0.1523,0.3,0.2428,0.1373)^{\mathrm{T}} \\
\omega^{3}=(0.1613,0.1323,0.2998,0.2455,0.1613)^{\mathrm{T}} \\
\omega^{4}=(0.1772,0.1250,0.2960,0.2515,0.1502)^{\mathrm{T}}
\end{gathered}
$$

(4) Receive time attribute $\varpi=(0.0403,0.0638,0.1231,0.7728)^{\mathrm{T}}$ based on (3), count mean $E_{i}=\sum_{k=1}^{4} w_{i}^{k} \varpi_{k}$ and variance $\sigma_{i}^{2}=\sum_{k=1}^{l}\left(w_{i}^{k}-E_{i}\right)^{2}$ of alternative $x_{i}$.

$$
\begin{gathered}
E_{1}=0.1727, E_{2}=0.1294, E_{3}=0.2969, E_{4=0.25,} E_{5}=0.1509 . \\
\sigma_{1}^{2}=0.00215, \sigma_{2}^{2}=0.00218, \sigma_{3}^{2}=0.00003, \sigma_{4}^{2}=0.00008, \sigma_{5}^{2}=0.00031 .
\end{gathered}
$$

(5) Rank mean and variance of five voyages.

Ordering with mean:

$$
E_{3}>E_{4}>E_{1}>E_{5}>E_{2}
$$

Ordering with variance:

$$
\sigma_{3}^{2}<\sigma_{4}^{2}<\sigma_{5}^{2}<\sigma_{1}^{2}<\sigma_{2}^{2}
$$

(6) Judge and analyze, confirm ordering result of alternatives.

There are some ordering position differences between mean and variance of $x_{1}$ and $x_{5}$. Based on decision maker's experiences, let $\xi=0.1, \xi \cdot \frac{1}{m}=0.1 \times \frac{1}{5}=0.02$, and $E_{1}-E_{5}=0.0218>0.02$. Finally, we choose ordering result with mean as ultimate result.

(7) Rank all the alternatives ${ }_{i}$, and we can see that the ranking order of five flight voyages is: 


$$
x_{3}>x_{4}>x_{1}>x_{5}>x_{2}
$$

Above all, it's difficult to judge some alternatives where means are similar in traditional dynamic decision making. In order to distinguish stand or fall, it introduces Mean-Variance statistics error multi-attribute dynamic decision making for resolving the intricate situation, and enhances the accuracy of decision result.

As shown in Figure. 3, the touchdown point of third voyage locates between the $2^{\text {nd }}$ cable and the $3^{\text {rd }}$ one, and we would call it optimal touchdown point. Other voyages touchdown points distributions are nearer the desired one, and the landing risk is decreased.

\section{Conclusion}

This paper has introduced an improved decision making approach based on MeanVariance statistics error multi-attribute dynamic decision making. Mean and variance would be synthesized to solve the shortage of traditional dynamic decision making, and the decision making problem whose rank alike especially. At last Mean-Variance statistics error multi-attribute dynamic decision making has an application in landing risk assessment of carrier-based aircraft. Simulation results show that the reasonable and application of the new algorithm.

\section{Acknowledgements}

The author would like to thank the anonymous referees for their valuable suggestions. This work was supported by the Natural Science Foundation of Heilongjiang Province of China (Grant Nos. F2015045).

\section{References}

[1] L Hui, J Hai-Tao, S Xiao-dong, "Modeling landing signal officer instruction associated with operation guide system", International Journal of Control and Automation, vol. 8, no. 2, (2015), pp. 373-382.

[2] L Hui, S Xiao-dong, J Hai-Tao, "Asymmetric variable universe adaptive landing fuzzy controller for carrier-based aircraft"t, International Journal of Smart Home, vol. 9, no. 5, (2015), pp. 31-40.

[3] L Hui, "Integrated Evaluation Technology of landing signal officer for carrier-based aircraft", International Journal of Multimedia and Ubiquitous Engineering, vol. 11, no. 1, (2016).

[4] J Hai-Tao, S Xiao-dong, L Hui, "Dynamic multi-attribute decision making based on advantage retention degree. International Journal of Control and Automation", vol. 7, no. 9, (2014), pp. 389-398.

[5] Z Shi-fang, "Application of some fuzzy multi-attribute decision making", Xi'an Electronic and Engineering University, Xi'an, (2012).

[6] J Hai-Tao, L Hui, SXiao-dong, "Landing risk assessment of carrier-based aircraft based on fuzzy multiattribute group decision making". International Journal of Control and Automation, vol. 9, no. 8, (2015).

[7] T Rudowsky, S Cook, Ml Hynes, etal. "Review of the carrier approach criteria for carrier-based aircraft". Techinical report NAWCADPAX/TR-2002/71, (2002).

[8] R K. Heffley, "Outer-loop control factor for carrier aircraft.USA". (1990)

[9] R A Hess. Simplified approach for modeling pilot pursuit control behaviour in multi-loop flight control taks[J]. Institution of mechanical engineer, vol. 220, no. 2, (2006), pp. 85-102.

[10] NAVAIR 00-80T-104. NATOPS Landing signal officer manual.US. (2001)

[11] Q Xiangju, C Hailiang, "Variable strategy pilot model of carrier landing approach. Journal of Beijing University of Aeronautics and Astronautics", vol. 29, no. 11, (2003) pp. 993-997.

[12] X Zeshui, D Qingli, "Multi-attribute decision making based on fuzzy linguistic assessments". Journal of Southeast University (Natural Science Edition), vol. 32, no. 4, (2002), pp. 1-3.

[13] H Zuosheng, L Peide. "Multiple attribute decision making method based on trapezoid fuzzy linguistic variables". Fuzzy Systems and Mathematics, vol. 25, no. 3, (2011), pp. 119-126.

[14] F. Herrera, L. Martinez, "An approach for combing numerical and linguistic information based on the 2-tuple fuzzy linguistic representation model in decision making". International Journal of Uncertainty, Fuzziness and Knowledge-Based Systems, no. 8, (2000) pp. 539-562.

[15] L D F, Y J B, "Fuzzy liner programming technique for multi-attribute group decision making in fuzzy environments. Information Sciences", (2004), pp. 158: 263-275.

[16] Z Xu, "Group decision making with triangular fuzzy linguistic variables" //IDEAL 2007, LNCS, (2007), 4881: pp. 17-26. 
[17] L Xuechun, C Senfa, "Multiple attribute decision making method based on trapezoid fuzzy linguistic variables". Journal of Southeast University(English Edition), vol. 24, no. 4, (2008), pp. 478-481.

[18] P Liu, s Yu, "The extended TOPSIS based on trapezoid fuzzy linguistic variables". Journal of Convergence Information Technology, vol. 5, no. 4, (2010), pp. 38-53.

[19] X Zeshui, "Study on methods for multiple attribute decision making under some situations". School of Economics \& Management, Southeast University, (2002)

[20] L Lin, C Yunxiang, G Zhihao, ,Approach to the multi-attribute linguistic decision making and its' application”. System Engineering and Electronics, vol. 31, no. 1, (2009) pp. 113-115, 207.

[21] Z Yingxin, "Analysis on mine coal dust explosion dangree base on normal fuzzy function". Coal Engineering, no. 7, (2009), pp. 72-74 .

[22] Lv Zehua, C Chuanbo, Q Peiyu, "Normal distribution fuzzy sets-A new extension of fuzzy sets",Computer Science, 2006, vol. 33, no. 11, (2006), pp. 1-14, 173.

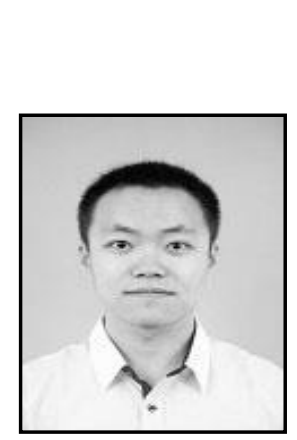

\section{Authors}

Hui Li, He received a D.E. degree in Control Theory and Control Engineering from Harbin Engineering University, Harbin, China, 2013. He is the member of council of the Operations Research Society of China. His recent research interests are in intelligent control, Multi-attribute decision making, fuzzy decision making. 
International Journal of Security and Its Applications

Vol. 10, No. 3 (2016) 